\title{
Ischio-Rectal Abscess Caused by Ingested Bone
}

\section{Shivakumar SP* and Shanmugam RP}

Department of Surgical Gastroenterology, Kumaran Hospital, Chennai, India

\author{
Abstract \\ Ischio-Rectal Abscess is rarely caused by ingested foreign body. We present a case of Ischio Rectal Abscess \\ caused by ingested bone.
}

Keywords: Ischio-rectal abscess; Foreign body; Ingested bone; Chicken bone; Peri anal abscess

\section{Introduction}

Anorectal sepsis is classified into perianal abscess which is the most common followed by Ischio Rectal Abscess. The incidence of ischio rectal abscess located around the anal canal in relation to the line joining both the ischeal tuberosity is $53 \%$ in posterior, $35 \%$ in lateral and $12 \%$ in anterior quadrant 2 .

\section{Case Report}

A 28 years old female presented with severe pain in the perianal region and mild grade fever for the past 1 week. Her bowel habits were normal. She has undergone laser haemorrhoidectomy 3 weeks earlier. No history of diabetes, hypertension, crohns, ulcerative colitis or tuberculosis. Blood investigation showed an elevated total count.

On general examination her vital signs were stable. Local examination revealed a warm and tender swelling around $2 \times 3 \mathrm{cms}$ in size in the left perianal region along 4 o clock position, $2.5 \mathrm{cms}$ from the anus.

Per rectal examination revealed a tender boggy swelling in the left lateral wall of rectum and anal canal. Under General anaesthesia a cruciate incision was made on the ischio rectal region and deepened on the left side cutting the skin and subcutaneous fat tissue. An incision and drainage was completed, letting out $30 \mathrm{ml}$ of pus [1]. Loculations were broken down. A thin $3 \mathrm{~cm}$ long bone piece was retrieved from the cavity (Figure 1). No internal opening into the lower rectum was made out. Culture from the pus showed no growth of any organism [1-3].

\section{Discussion}

The main causes are of perianal abscess are diabetes, crohns disease,

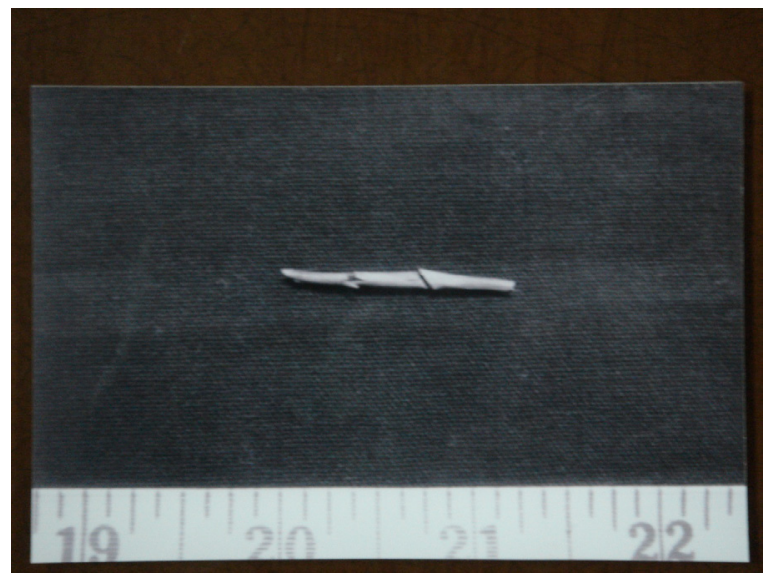

Figure 1: $A$ thin $3 \mathrm{~cm}$ long bone piece was retrieved from the cavity. ulcerative colitis, pregnancy and rarely foreign body. The majority of the foreign bodies are inserted via the anal canal. Occasionally some objects become arrested in the anorectal region following ingestion, most commonly animal bones associated with food ingestion as it has happened in our case. Ingestion of foreign bodies is more common in children, alcoholics, denture wearers and those who are mentally impaired. Although 10-20\% 3 of ingested foreign bodies fail to pass through the entire gastrointestinal tract. Less than $5 \%$ cause perforation.

The potential sites of arrest include the oesophagus, pylorus, ileocecal valve (which is the commonest site of perforation [4], colon and the anus. Less than $5 \%$ of patients require surgery. Some can breach the gut wall introducing perianal sepsis. Foreign bodies that lodge more proximally in the rectum might go unnoticed until perforation and sepsis occur. A supralevator abscess may then extend into the ischiorectal space and clinically present as an ischiorectal abscess [5], which could have occurred in our patient.

The pathogenesis of ischorectal abscess is variable. In some patients the intermuscular collection extends laterally through the conjoined longitudinal muscle and through the lower fibres of the external anal sphincter into the ischiorectal fossa. In others, the infection extends caudally in the intermuscular plane and then around the lower border of the external anal sphincter into the ischiorectal fossa. In a few patients a supralevator abscess may burst through the fibres of the puborectalis into the ischiorectal fossa [6]. Finally, the ischiorectal fossa may become infected following an episode of septicaemia from a distant focus.

Clinical features of isciorectal abscess are less well defined. Fever is more common and associated with constitutional disturbance. The swelling is much more diffuse, and tends to involve the entire ischiorectal region. Examination reveals a vague area of swelling beside the rectum but bulging into the anal canal is unusual. Bilateral involvement to form a horseshoe abscess occurs by posterior spread into the ischiorectal fossa of the opposite side. There may be considerable tissue necrosis due to pus under tension in the relatively avascular ischiorectal space. Sometimes a collection high in the ischiorectal fossa may be associated with no clinical signs at all [4-6].

These patients need a careful examination, including proctosigmoidoscopy and fistulogram, to identify the presence of an

${ }^{*}$ Corresponding author: Dr. SP Shiva Kumar, Department of Surgical Gastroenterology, Kumaran Hospital, Chennai-600010, India, E-mail: drshiv74@hotmail.com

Received June 23, 2013; Accepted July 18, 2013; Published July 20, 2013

Citation: Shivakumar SP, Shanmugam RP (2013) Ischio-Rectal Abscess Caused by Ingested Bone. J Clin Case Rep 3: 293. doi: 10.4172/2165-7920.1000293

Copyright: (C) 2013 Shivakumar SP, et al. This is an open-access article distributed under the terms of the Creative Commons Attribution License, which permits unrestricted use, distribution, and reproduction in any medium, provided the original author and source are credited. 
internal opening and to ascertain the extent of the infective process, particularly any supralevator or intersphinecteric component.

A soft malleable blunt probe may be associated with the formation of a false passage, particularly in the presence of acute sepsis. A soft ureteric catheter is probably safer for identifying an internal opening.If pus culture reveals Staph. aureus, a second EUA is probably unnecessary since the chance of a missed internal opening is then remote. If cultures reveal E. Coli Proteus spp., Klbesiella spp. or gut-specific bacteriodes, there is a strong possibility of a fistula. Intrarectal ultrasound often failed to image the internal opening and the anatomy of the abscess in relation to external sphincter. MRI is the investigation of choice in all complex anorectal sepsis.

Ischiorectal sepsis may progress if not treated adequately to spreading extraperitoneal cellulitis which can be fatal, particularly in diabetic or immunocompromised patients. Recurrent abscess or fistula was reported in $65 \%$ of patients when the abscess was merely drained, whereas $31 \%$ had a recurrent abscess when fistulotomy was performed at the time of drainage [7]. Recurrence occurred in only $3 \%$ of patients managed initially by drainage alone, followed later by a staged fistulotomy. Hence immediate drainage with staged fistulotomy or seton insertion, for high fistula results in fewer recurrence and incontinence compared to drainage alone.

Recurrence has been attributed to: (1) failure to identify an internal opening (2) inadequate assessment of the extent of the abscess, resulting in incomplete drainage; (3) spontaneous rupture of the abscess so that a proper examination under anaesthetic was never performed; and (4) the presence of an underlying disorder, such as Crohn's disease, AIDS or tuberculosis [7].
Antibiotics are used during surgical drainage and in patients with valvular disease of the heart, those with a prosthetic implant and in diabetics. Primary suture, curettage or the uses of drainage tubes are not recommended, for fear of creating a high fistula and recurrence. If a supralevator abscess is due to an upward extension of a Transsphincteric fistula complicating an ischiorectal abscess, it should never be drained into the rectum since this will almost invariably create an extransphincteric fistula. These cases must be treated by dependent perineal drainage through the ischiorectal fossa. At a later stage the lower fibres of the external sphincter may be laid open to treat the transphincteric fistula $[6,7]$.

\section{References}

1. Ramanujam PS, Prasad ML, Abcarian H, Tan AB (1984) Perianal abscesses and fistulas. A study of 1023 patients. Dis Colon Rectum 27: 593-597.

2. Barone JE, Yee J, Nealon TF Jr (1983) Management of foreign bodies and trauma of the rectum. Surg Gynecol Obstet 156: 453-457.

3. Velitchkov NG, Grigorov GI, Losanoff JE, Kjossev KT (1996) Ingested foreign bodies of the gastrointestinal tract: retrospective analysis of 542 cases. World J Surg 20: 1001-1015.

4. Davies DH (1991) A chicken bone in the rectum. Arch Emerg Med 8: 62-64.

5. Hacker JF 3rd, Cattau EL Jr (1986) Management of gastrointestinal foreign bodies. Am Fam Physician 34: 101-108.

6. Held D, Khubchandani I, Sheets J, Stasik J, Rosen L, et al. (1986) Management of anorectal horseshoe abscess and fistula. Dis Colon Rectum 29: 793-797.

7. Chrabot CM, Prasad ML, Abcarian H (1983) Recurrent anorectal abscesses. Dis Colon Rectum 26: 105-108. 